\title{
Crystal Structure and Thermal Stability Study on Tetrabutylammonium Hexamolybdate $\left[n-\mathrm{Bu}_{4} \mathrm{~N}\right]_{2}\left[\mathrm{Mo}_{6} \mathrm{O}_{19}\right]$ (TBAM)
}

\author{
Pu Su Zhao ${ }^{\ddagger}$, Zhan Ru Zhao, Fang Fang Jian*, and Lu De Lu ${ }^{\dagger}$ \\ New Materials \& Function Coordination Chemistry Laboratory, Qingdao University \\ of Science and Technology, Qingdao, 266042, P. R. China \\ ${ }^{+}$Materials Chemistry Lahoratory; Nanjing University of Science and Technology, Nanjing, 210094, P. R. China
}

(2003.8.5 접수)

\section{Crystal Structure and Thermal Stability Study on Tetrabutylammonium Hexamolybdate $\left[n-\mathrm{Bu}_{4} \mathrm{~N}_{2}\left[\mathrm{Mo}_{6} \mathrm{O}_{19}\right](\mathrm{TBAM})\right.$}

\author{
Pu Su Zhao ${ }^{*}$ Zhan Ru Zhao, Fang Fang Jian*, and Lu De $\mathrm{Lu}^{\dagger}$ \\ New Materials \& Finction Coordination Chemistry Laboraton. Qingdao University \\ of Science and Technology, Qingdao, 266(142, P. R. China \\ 'Materials Chemistry Lahoratory. Nanjing University of Science and Techolog: Nanjing, 210094. P. R. China
}

(Received August 5, 2003)

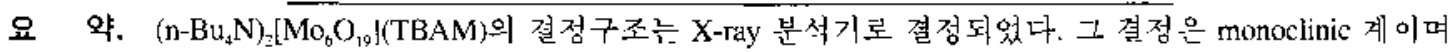
$\alpha=16.314(5), b=17.288(5), c=17.776(4) \AA A, \beta=101.47(3)$ 그리고s $x=4$ 의 결정파라미터를 갖는 sapce group 이 $C 2 / c$ 인 결정이다 $\left[\mathrm{Mo}_{6} \mathrm{O}_{9}\right]^{2-}$ 음이온에서, $\mathrm{Mo}$ 원자는 판면체의 여섯 개 모서리를 차지하며 가 $\mathrm{Mo}$ 원자는 여석개의 산슨원자에

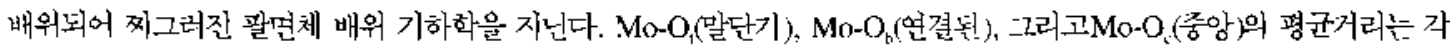
각 $1.680 \AA, 1.931 \AA$ 및 $2.325 \AA$ 이다. [n-Bu丶 $\mathrm{N}^{+}$양이온에서 N원자는 약간 찌]그려잔 사면체 모형올 갖는다. 격자안에 서 폭 넓은 $\mathrm{C}-\mathrm{H} \cdots \mathrm{O}$ 수소결합이 있으며, 그것에 의하여 분자늘을 연결하고 결정구조를 안정화 한다. 열눈서에 의하여 세목의 역적분핸는 두개의 전이가 일어나며 356.0 와 $803.5^{\circ} \mathrm{C}$ 에서 각각 무게를 잃는다. 그리고 분해돤 생성불은 $\mathrm{Mo}_{2} \mathrm{O}_{2}$ 로 추점된다. 따라서. 제목의 화합꿀은 높은 열적 안정성을 갖는다.

주제어: 이이소폴리옥손뎨이트, 결성구조, 열적 안정성

\begin{abstract}
The crystal structure of $\left[n-\mathrm{Bu}_{4} \mathrm{~N}_{2}\left[\mathrm{Mo}_{6} \mathrm{O}_{19}\right](\mathrm{TBAM})\left(n-\mathrm{Bu} u_{4} \mathrm{~N}=\right.\right.$ tetrabutylammonium) has been determined by X-ray crystallography. It crystallizes in the monoclinic system, space group $C 2 / c$, with lattice parameters $a=$ $16.314(5), b=17.288(5), c=17.776(4) \AA, b=101.47(3)$, and $Z=4$. In $\left.\mid \mathrm{Mo}_{6} \mathrm{O}_{19}\right]^{2-}$ anion. Mo atoms occupy six vertices of octahedron and each Mo atom is coordinated by six oxygen atoms to adopt distorted octahedral coordination geometry. The average bond distance of $\mathrm{Mo}-\mathrm{O}_{\mathrm{t}}$ (terminal), $\mathrm{Mo}-\mathrm{O}_{\mathrm{b}}$ (bridged) and $\mathrm{Mo}-\mathrm{O}_{\mathrm{c}}$ (central) are $1.680 \AA, 1.931 \AA$, and $2.325 \AA$. respectively. In $\left[n-\mathrm{Bu}_{4} \mathrm{~N}\right]^{+}$cation, the $\mathrm{N}$ alom possesses a slightly distorted tetrahedral geomery. There are some potential extensive $\mathrm{C}-\mathrm{H} \ldots \mathrm{O}$ hydrogen bonds in the lattice, by which connecte molecules and stabilize the crystal structure. Thermogravimetric analysis suggests that thermal decomposition of the title compound includes two transitions and it loses weight at 356.0 and $803.5^{\circ} \mathrm{C}$, respectively, and the residue presumable be $\mathrm{Mo}_{2} \mathrm{O}_{2}$. Accordingly, the title compond has high thermal stability.
\end{abstract}

Keywords: Isopolyoxomolybdate. Tetrabutylammonium, Crystal Structure, Thermalstability 


\section{INTRODUCTION}

It is well known that polyoxoanions conjugated with organic molecules have the abilitiy for photochromism, and that photochromism involving these compounds is truly reversible.$^{1.7}$ In order to prepare reversible photochromism materials, photosensitive organic molecules as donor and Type I polyoxometalate anions having easily reduced properties ${ }^{8}$ as acceptor have been adopted. Such anions are still of interest because of their high electron acceptor capability. ${ }^{9} \mathrm{Xu}$ et al has reported reversible photochromism charge-transfer salt using photosensitive tetrabutylammonium (TBA) as donor and the hexamolybdate dianion $\left(\mathrm{Mo}_{6} \mathrm{Ol}_{9}^{2-}\right)$ as acceptor under ultraviolet irradiation. ${ }^{10}$ However, the crystal structure of tetrabutylammonium hexamolybdate $\left[n-\mathrm{Bu}_{4} \mathrm{~N}\right]_{2}$ $\left[\mathrm{Mo}_{6} \mathrm{O}_{14}\right]$ (TBAM) has never been reported. In this paper, we report the crystal structure of TBAM, and the thermal stability of it.

\section{EXPERAMENT SECTION}

Hydrothermal synthesis of TBAM. An acetonitrile solution of the tetrabutylammonium bromide (TBABr) and isopolyoxomolybdate $\mathrm{Mo}_{6} \mathrm{O}_{19}^{2-}$ ane mixed with stirring and its $\mathrm{pH}$ value was adjusted to 6 with dilution $\mathrm{HCl}$, then the mixture was sealed in a $25 \mathrm{~mL}$ stainless-steel reactor with Teflon liner at $100^{\circ} \mathrm{C}$ for $72 \mathrm{~h}$, resulting in the formation of the light blue crystals of the title complex. Yield: $85 \%$. Calc. for $\mathrm{C}_{32} \mathrm{H}_{72} \mathrm{Mo}_{6} \mathrm{~N}_{2} \mathrm{O}_{19}: \mathrm{C}, 28.16 \% ; \mathrm{H}, 5.31 \% ; \mathrm{N}, 2.05 \%$. Anal. Found: C, $28.01 \% ; \mathrm{H}, 4.98 \% ; \mathrm{N}, 1.97 \%$.

$\mathrm{X}$-ray structure determination. The selected crystal of $\left[n-\mathrm{Bu}_{4} \mathrm{~N}_{2}\left[\mathrm{Mo}_{h} \mathrm{O}_{19}\right]\right.$ was mounted on an Rigaku Raxis-IV diffractometer. Reflection data were measured at $293 \mathrm{~K}$, using graphite monochromated $\mathrm{M}_{0}$ $\mathrm{K}_{\mathrm{rx}}(\lambda=0.71073 \AA)$ radiation $\omega$ scan mode. Intensities were corrected for Lorentz and polarization effects and empirical absorption, and the data reduction using SADABS program. ${ }^{11}$ The structure were solved by direct methods using SHELX-97.'2 All the nonhydrogen atoms were refined on $F^{2}$ anistropically by full-matrix least squares method. The hydrogen atom positions were fixed geometri-
Table 1. Sumnary of Crystallographic Results for $\left[n-\mathrm{Bu}_{4} \mathrm{~N}\right]_{2}$ $\left|\mathrm{Mo}_{h} \mathrm{O}_{l s}\right|$

\begin{tabular}{|c|c|}
\hline Color/shape & light blue/prism \\
\hline Empirical formula & $\mathrm{C}_{32} \mathrm{H}_{32} \mathrm{Mo}_{6} \mathrm{~N}_{2} \mathrm{O}_{10}$ \\
\hline Formula weight & 1364.56 \\
\hline Crystal system & Monoclinic \\
\hline Space group & $C 2 / c$ \\
\hline \multicolumn{2}{|l|}{ Unit cell dimensions } \\
\hline$a, \AA$ & $16.314(5)$ \\
\hline$b, \AA$ & $17.288(5)$ \\
\hline$c, \AA$ & $17.776(4)$ \\
\hline$b{ }^{\circ}$ & $101.47(3)$ \\
\hline Cell volume. $\mathrm{A}^{3}$ & $4913(2)$ \\
\hline$Z$ & 4 \\
\hline$D$ (calcd.) $\mathrm{Mg} / \mathrm{m}^{3}$ & 1.845 \\
\hline Abs coeff. $\mathrm{mm}^{-1}$ & 1.552 \\
\hline$F(000)$ & 2728 \\
\hline Radiation & Mo $K \alpha(\lambda=0.071073 \AA)$ \\
\hline Temp. K & $293(2)$ \\
\hline$\theta$ range. ${ }^{\circ}$ & 1.73 to 27.48 \\
\hline Limitting indices & $-15 \leq \mathrm{k} \leq 20,-14 \leq \mathrm{k} \leq 22,-22 \leq \mathrm{l} \leq 20$ \\
\hline Reflections collected & 6760 \\
\hline Independent reflections & $4144\left[K_{\text {(Lut })}=0.02991\right.$ \\
\hline$R[I>2 \sigma(I)]$ & $R I=0.0334, w R 2=0.0563$ \\
\hline$R$ (all data) & $R 1=0.1010, w R 2=0.0641$ \\
\hline Goodness-of-fit on $F^{2}$ & 0.898 \\
\hline Larges1 diff. peak and hole & 0.490 and $-0.420 \mathrm{eA}^{-3}$ \\
\hline
\end{tabular}

cally at calculated distances and allowed to ride on the parent carbon atoms. The contributions of these hydrogen atoms were included in structure-factor calculations. The final least-square cycle gave $R=$ $0.0334, R_{\mathrm{w}}=0.0563$ for 2572 reflections with $I>2 \sigma$ $(f)$; the weighting scheme, $w=1 /\left[\sigma^{2}\left(F_{o}^{2}\right)+(0.0259 P)^{2}\right.$ $+0.0000 P$ ], where $\left.P=\left(F_{r}^{2}\right)+2 F_{c}^{2}\right) / 3$. Atomic scattering factors and anomalous dispersion comections were taken from International Table for X-Ray Crystallography. ${ }^{13}$ A summary of the key crystallographic information is given in Table 1. The final position parameters of nonhydrogen atoms are given in Table 2 . Selected bond lengths ( $(\AA)$, possible hydrogen bonds $(\AA)$ and bond angles $\left({ }^{\circ}\right)$ are presented in Table 3, respectively.

\section{RESULT AND DISCUSSION}

Structure. The crystat structure of the complex $\left[n-\mathrm{Bu}_{4} \mathrm{~N}_{2}\left[\mathrm{Mo}_{6} \mathrm{O}_{19}\right]\right.$ consists of a symmetry $\left[\mathrm{Mo}_{6} \mathrm{O}_{19}\right]^{2-}$ 
Table 2. Alonic coordinates $\left(\times 10^{4}\right)$ and equivalent isotropic displacement parameters $\left(\AA^{2} \times 10^{3}\right), U_{i: 4:}$ is defined as one thied of the trace of the orthogonalized $U_{2}$ tenswr.

\begin{tabular}{|c|c|c|c|c|}
\hline atom & $x$ & $y$ & $z$ & $U_{e 4}$ \\
\hline $\operatorname{Mo}(1)$ & $3689(1)$ & $2892(1)$ & $1705(1)$ & $51(1)$ \\
\hline $\operatorname{Mo}(2)$ & 5000 & $1544(1)$ & 2500 & $46(1)$ \\
\hline $\operatorname{Mo}(3)$ & 5000 & $4243(1)$ & 2500 & $59(1)$ \\
\hline $\operatorname{Mo}(4)$ & $5620(1)$ & $2896(1)$ & $1431(I)$ & $51(1)$ \\
\hline $\mathrm{O}(1)$ & $2747(2)$ & $2870(2)$ & $1122(2)$ & $73(1)$ \\
\hline$O(2)$ & $3935(2)$ & $3985(2)$ & $1843(2)$ & $60(1)$ \\
\hline$O(3)$ & $4453(2)$ & $2901(2)$ & $992(2)$ & $55(1)$ \\
\hline$O(4)$ & $3944(2)$ & $1799(2)$ & $1848(2)$ & $50(1)$ \\
\hline$O(5)$ & 5000 & $2900(3)$ & 2500 & $37(1)$ \\
\hline$O(6)$ & $3441(2)$ & $2896(2)$ & $271 \mathrm{I}(2)$ & $53(1)$ \\
\hline$O(7)$ & 5000 & $566(3)$ & 2500 & $70(2)$ \\
\hline$O(8)$ & $5512(2)$ & $1808(2)$ & $1644(2)$ & $50(1)$ \\
\hline $\mathrm{O}(9)$ & $5507(2)$ & $3988(2)$ & $1646(2)$ & $58(1)$ \\
\hline$O(10)$ & 5000 & $5215(3)$ & 2500 & $85(2)$ \\
\hline$O(11)$ & $6084(2)$ & $2918(2)$ & $672(2)$ & $71(1)$ \\
\hline$N(1)$ & $2470(2)$ & $141(2)$ & $5 ! 6(2)$ & $49(1)$ \\
\hline$C(1)$ & $3812(5)$ & $-1269(6)$ & $2430(5)$ & $115(3)$ \\
\hline$C(2)$ & $3178(4)$ & $-705(4)$ & $2571(3)$ & $74(2)$ \\
\hline$C(3)$ & $2540(4)$ & $-492(4)$ & $1853(3)$ & $65(2)$ \\
\hline$C(4)$ & $2930(3)$ & $57(3)$ & $-1353(2)$ & $50(1)$ \\
\hline$C(5)$ & $5033(3)$ & $1215(4)$ & $-345(3)$ & $68(2)$ \\
\hline$C(6)$ & $4132(3)$ & $1361(3)$ & $-267(3)$ & $53(1)$ \\
\hline$C(7)$ & $3787(3)$ & $672(3)$ & $85(3)$ & $57(2)$ \\
\hline$C(8)$ & $2877(3)$ & $795(3)$ & $156(3)$ & $49(1)$ \\
\hline$C(9)$ & $2115(5)$ & $-1428(6)$ & $-1946(4)$ & $110(3)$ \\
\hline$C(10)$ & $2380(5)$ & $-1404(4)$ & $-1098(4)$ & $84(2)$ \\
\hline$C(11)$ & $2211(4)$ & $-629(4)$ & $-768(3)$ & $72(2)$ \\
\hline$C(12)$ & $2535(3)$ & $-623(3)$ & $104(3)$ & $59(2)$ \\
\hline$C(13)$ & $180(5)$ & $1784(4)$ & $1268(5)$ & $97(2)$ \\
\hline$C(14)$ & $413(4)$ & $1113(4)$ & $826(4)$ & $77(2)$ \\
\hline$C(15)$ & $1.346(3)$ & $1033(4)$ & $882(3)$ & $67(2)$ \\
\hline$C(16)$ & $1550(3)$ & $323(3)$ & $463(3)$ & $57(2)$ \\
\hline
\end{tabular}

anion and two $\left[n-\mathrm{Bu}_{4} \mathrm{~N}\right]^{-}$cations. Fig. 1 shows a perspective view of the title compound with atomic numbering scheme, and Fig. 2 shows a perspective view of the crystal packing in the unit cell.

In $\left[\mathrm{M}_{6} \mathrm{O}_{14}\right]^{2-}$ anion, six Mo atoms locate at the six vertexes of slightly distorted octahedron and nineteen oxygen atoms are divided into threc calcgories, with one oxygen atom lying in the central of above octahedron $\left(\mathrm{O}_{c}\right)$, six oxygen atoms occupying the terminal positions of above octahedron $\left(\mathrm{O}_{1}\right)$ and twelve oxygen as bridged atoms $\left(\mathrm{O}_{\mathrm{h}}\right)$ bridging six Mo atoms, respectively. All the angels of $\mathrm{Mo}_{-}-\mathrm{O}_{-}-\mathrm{Mo}$ are nearly $90^{\circ}$ or $180^{\circ}$. Each Mo atom possesses a distoned octahedral coordination geometry, which is coordinated by six oxygen atoms with the central atom and one terminal oxygen atom in axial position, and four bridged oxygen atoms occupying equatorial position. As seen from the Table 3 , the trans bond angles forming by terminal oxygen atom, Mo atom and central oxygen atom are close to $180^{\circ}$, and the cis angles of $\mathrm{O}-\mathrm{Mo}-\mathrm{O}$ are nearly to $90^{\circ}$. Because of the existence of bridged oxygen atoms, the angles of opposite $\mathrm{O}_{\mathrm{n}}-\mathrm{Mo}-\mathrm{O}_{b}$ are all smaller than $180^{\circ}$ with average $\mathrm{O}_{\mathrm{b}}-\mathrm{Mo}-\mathrm{O}_{\mathrm{b}}$ about $153^{\circ}$. The average bond distance of $\mathrm{Mo}-\mathrm{O}, 1.680 \AA$ equates to that in TPPM [TPPM=bis(2,4,6-triphenylpyryllium) hexamolybdate], the distance $\mathrm{Mo}_{0} \mathrm{O}_{b} 1.931 \AA$ and Mo$\mathrm{O}_{\mathrm{c}} 2.325 \AA$ are longer than those in TPPM [ $\mathrm{Mo}-\mathrm{O}_{\mathrm{b}}$ $1.917 \AA$ and $\mathrm{Mo}-\mathrm{O}, 2.312 \AA]^{10}$. But all these values are in the range of with those reported previously. ${ }^{1+15}$ In the slight distorted octahedral geometry of $\left[\mathrm{Mo}_{n} \mathrm{O}_{19}\right]^{2-}$ anion, three $\sigma_{\mathrm{n}}$ symmetrical planes were occupied by thrce molecular planes, each of which contained thirteen atoms (four $\mathrm{Mo}$ atoms, four $\mathrm{O}_{\mathrm{b}}$ atoms, four $\mathrm{O}_{\mathrm{t}}$ atoms and one $\mathrm{O}_{c}$ atom) and the largest deviations of which are $0.012,0.045$ and $0.016 \dot{A}$, respectively. The above threc molecular planes are almost vertical each other, with the dihedral 89.82 , 89.90 and $89.92^{\circ}$, respectively.

In the $\left[\mathrm{n}-\mathrm{Bu}_{\star} \mathrm{N}\right]^{+}$cation, the $\mathrm{N}$ atom adopts a slightly distorted tetrahedral geometry with the $\mathrm{C}-\mathrm{N}-\mathrm{C}$ bond angles ranging from $108.1^{\circ}$ to $111.5^{\circ}$. The $\mathrm{C}-\mathrm{N}$ and $\mathrm{C}-\mathrm{C}$ bond lengths fall within the normal rang.

There arc some potentially weak ( $\mathrm{C}-\mathrm{H} \cdots \mathrm{Y}$ hydrogen bonds. $\mathrm{Y}=\mathrm{O})$ interactions in the lattice. ${ }^{16-17}$ The $\mathrm{O}(3)$ atom with $\mathrm{C}(6)$ atom in $\left[\mathrm{n}-\mathrm{Bu}_{4} \mathrm{~N}\right]^{+}$cation forms potentially weak $\mathrm{C}-\mathrm{H}$... O intramolecular interactions, the donor and acceptor distance is $3.4435 \AA$ for $\mathrm{C}(6)-\mathrm{H}(6 \mathrm{~A}) \cdots \mathrm{O}(3)$. The $\mathrm{O}(1)$ atom with $\mathrm{C}(8)$ atom forms $\mathrm{C}-\mathrm{H} \cdots \mathrm{O}$ intermolecular interactions, the donor and acceptor distance being $3.2600 \AA$ for $\mathrm{C}(8)-\mathrm{H}(8 \mathrm{~B}) \cdots \mathrm{O}(1)$ (symmetry code: $1 / 2-x, 1 / 2-y,-z$ ). The bond angles of $\mathrm{C}(6)-\mathrm{H}(6 \mathrm{~A}) \cdots \mathrm{O}(3)$ and $\mathrm{C}(8)-\mathrm{H}(8 \mathrm{~B})$ $\cdots O(1)$ are $164.86^{11}$ and $131.18^{\circ}$, respectively. In the solid state, these interactions together with elecrrostatic forces connected molecules and stabilize the crystal structures. 
Pu Su Zhao, Zhan Ru Zhao, Fang Fang Jian and Lu De Lu

Table 3. Selected bond lengths $(\AA)$ and bond angles $\left({ }^{\circ}\right)$ of the title compound

\begin{tabular}{|c|c|c|c|}
\hline $\mathrm{Mo}(1)-\mathrm{O}(1)$ & $1.674(3)$ & $\operatorname{Mo}(1)-O(6)$ & $1.910(3)$ \\
\hline $\mathrm{Mo}(1)-\mathrm{O}(2)$ & $1.937(3)$ & $\mathrm{Mo}(1)-\mathrm{O}(4)$ & $1.942(3)$ \\
\hline $\mathrm{Mo}(\mathrm{J})-\mathrm{O}(3)$ & $1.945(3)$ & $\operatorname{Mo}(1)-O(5)$ & $2.3177(10)$ \\
\hline $\operatorname{Mo}(2)-O(7)$ & $1.690(5)$ & $\mathrm{Mo}(2)-\mathrm{O}(4) \# 1$ & $1.926(3)$ \\
\hline $\mathrm{Mo}(2)-\mathrm{O}(4)$ & $1.927(3)$ & $\mathrm{Mo}(2)-\mathrm{O}(8) \# 1$ & $1.929(3)$ \\
\hline $\mathrm{Mo}(2)-\mathrm{O}(8)$ & $1.932(3)$ & $\operatorname{Mo}(2)-O(5)$ & $2.345(5)$ \\
\hline $\operatorname{Mo}(3)-O(10)$ & $1.680(5)$ & $\mathrm{Mo}(3)-\mathrm{O}(9)$ & $1.918(3)$ \\
\hline $\mathrm{Mo}(3)-\mathrm{O}(9) \# 1$ & $1.921(3)$ & $\operatorname{Mo}(3) \cdot O(2)$ & $1.942(3)$ \\
\hline $\mathrm{Mo}(3)-\mathrm{O}(2) \# 1$ & $1.943(3)$ & $\mathrm{M}(3)-\mathrm{O}(5)$ & $2.321(5)$ \\
\hline $\mathrm{Mo}(4)-\mathrm{O}(11)$ & $1.675(3)$ & $\operatorname{Mo}(4)-O(3)$ & $1.909(3)$ \\
\hline $\mathrm{Mo}(4)-\mathrm{O}(8)$ & $1.935(3)$ & $\operatorname{Mo}(4)-O(6) \#]$ & $1.936(3)$ \\
\hline $\operatorname{Mo}(4)-O(9)$ & $1.939(3)$ & $\operatorname{Mo}(4)-O(5)$ & $2.3228(7)$ \\
\hline $\mathrm{O}(5)-\mathrm{Mo}(1) \# 1$ & $2.3177(10)$ & $\mathrm{O}(5)-\mathrm{Mo}(4) \# 1$ & $2.3229(7)$ \\
\hline $\mathrm{O}(6)-\mathrm{Mo}(4) \# \mathrm{l}$ & $1.936(3)$ & & \\
\hline $\mathrm{O}(1)-\mathrm{MO}(1)-\mathrm{O}(6)$ & $103.90(15)$ & $\mathrm{O}(1)-\mathrm{Mo}(1)-\mathrm{O}(2)$ & $103.94(17)$ \\
\hline $\mathrm{O}(6)-\mathrm{Mo}(1)-\mathrm{O}(2)$ & $87.60(14)$ & $\mathrm{O}(1)-\mathrm{Mo}(1)-\mathrm{O}(4)$ & $101.96(17)$ \\
\hline $\mathrm{O}(6)-\mathrm{Mo}(1)-\mathrm{O}(4)$ & $87.55(14)$ & $\mathrm{O}(2)-\mathrm{Mo}(1)-\mathrm{O}(4)$ & $354.06(12)$ \\
\hline $\mathrm{O}(1)-\mathrm{M} O(1)-\mathrm{O}(3)$ & $102.99(15)$ & $\mathrm{O}(6)-\mathrm{M} \propto(1)-\mathrm{O}(3)$ & $153.10(11)$ \\
\hline $\mathrm{O}(2)-\mathrm{Mo}(1)-\mathrm{O}(3)$ & $85.92(15)$ & $\mathrm{O}(4)-\mathrm{Mo}(1)-\mathrm{O}(3)$ & $86.98(14)$ \\
\hline $\mathrm{O}(1)-\mathrm{Mo}(1)-\mathrm{O}(5)$ & $178.85(17)$ & $\mathrm{O}(6)-\mathrm{Mo}(1)-\mathrm{O}(5)$ & $76.75(9)$ \\
\hline $\mathrm{O}(2)-\mathrm{Mo}(\mathrm{I})-\mathrm{O}(5)$ & $76.99(15)$ & $\mathrm{O}(4)-\mathrm{Mo}(1)-\mathrm{O}(5)$ & $77.09(15)$ \\
\hline $\mathrm{O}(3)-\mathrm{Mo}(1)-\mathrm{O}(5)$ & $76.35(8)$ & $\mathrm{O}(7)-\mathrm{Mo}(2)-\mathrm{O}(4) \# !$ & $103.15(10)$ \\
\hline$O(7)-\mathrm{Mo}(2)-\mathrm{O}(4)$ & $103.30(10)$ & $\mathrm{O}(4) \# 1-\mathrm{Mo}(2)-\mathrm{O}(4)$ & $153.54(19)$ \\
\hline $\mathrm{O}(7)-\mathrm{Mo}(2)-\mathrm{O}(8) \# 1$ & $103.52(10)$ & $\mathrm{O}(4) \# 1-\mathrm{Mo}(2)-\mathrm{O}(8) \# 1$ & $87.02(14)$ \\
\hline $\mathrm{O}(4)-\mathrm{Mo}(2)-\mathrm{O}(8) \# 1$ & $86.85(13)$ & $\mathrm{O}(7)-\mathrm{Mo}(2)-\mathrm{O}(8)$ & $103.87(10)$ \\
\hline $\mathrm{O}(4) \# 1-\mathrm{Mo}(2)-\mathrm{O}(8)$ & $86.80(13)$ & $\mathrm{O}(4)-\mathrm{Mo}(2)-\mathrm{O}(8)$ & $86.91(14)$ \\
\hline $\mathrm{O}(8) \# \mathrm{I}-\mathrm{M}$ o(2)-O(8) & $152.61(19)$ & $\mathrm{O}(7)-\mathrm{Mo}(2)-\mathrm{O}(5)$ & 180.0 \\
\hline $\mathrm{O}(4) \# 1-\mathrm{Mo}(2)-\mathrm{O}(5)$ & $76.85(10)$ & $\mathrm{O}(4)-\mathrm{Mo}(2)-\mathrm{O}(5)$ & $76.70(10)$ \\
\hline $\mathrm{O}(8) \# 1-\mathrm{Mo}(2)-\mathrm{O}(5)$ & $76.48(10)$ & $\mathrm{O}(8)-\mathrm{Mo}(2)-\mathrm{O}(5)$ & $76.13(10)$ \\
\hline $\mathrm{O}(10)-\mathrm{Mo}(3)-\mathrm{O}(9)$ & $103.12(10)$ & $\mathrm{O}(10)-\mathrm{Mo}(3)-\mathrm{O}(9) \# 1$ & $103.46(10)$ \\
\hline $\mathrm{O}(9)-\mathrm{Mo}(3)-\mathrm{O}(9)+1$ & $153.4(2)$ & $O(10)-\operatorname{Mo}(3)-\mathrm{O}(2)$ & $103.19(10)$ \\
\hline $\mathrm{O}(9)-\mathrm{Mo}(3)-\mathrm{O}(2)$ & $86.96(14)$ & $\mathrm{O}(9) \# 1-\mathrm{Mo}(3)-\mathrm{O}(2)$ & $87.03(14)$ \\
\hline $\mathrm{O}(10)-\mathrm{Mo}(3)-\mathrm{O}(2) \# 1$ & $103.35(10)$ & $\mathrm{O}(9)-\mathrm{Mo}(3)-\mathrm{O}(2) \# \mathrm{I}$ & $87.07(14)$ \\
\hline $\mathrm{O}(9) \# 1-\mathrm{Mo}(3)-\mathrm{O}(2) \# 1$ & $86.84(14)$ & $\mathrm{O}(2)-\mathrm{Mo}(3)-\mathrm{O}(2) \# 1$ & $153.46(19)$ \\
\hline $\mathrm{O}(10)-\mathrm{Mo}(3)-\mathrm{O}(5)$ & $180.000(1)$ & $\mathrm{O}(9)-\mathrm{Mo}(3)-\mathrm{O}(5)$ & $76.88(10)$ \\
\hline $\mathrm{O}(9) \# \mathrm{I}-\mathrm{Mo}(3)-\mathrm{O}(5)$ & $76.54(10)$ & $\mathrm{O}(2)-\mathrm{Mo}(3)-\mathrm{O}(5)$ & $76.81(10)$ \\
\hline $\mathrm{O}(2) \# 1-\mathrm{Mo}(3)-\mathrm{O}(5)$ & $76.65(10)$ & $\mathrm{O}(11)-\mathrm{Mo}(4)-\mathrm{O}(3)$ & $104.18(15)$ \\
\hline $\mathrm{O}(11)-\mathrm{Mo}(4)-\mathrm{O}(8)$ & $104.82(17)$ & $\mathrm{O}(3)-\mathrm{Mo}(4)-\mathrm{O}(8)$ & $87.83(15)$ \\
\hline $\mathrm{O}(11)-\mathrm{Mo}(4)-\mathrm{O}(6) \# 1$ & $102.77(15)$ & $\mathrm{O}(3)-\mathrm{Mo}(4)-\mathrm{O}(6) \# \mathrm{l}$ & $153.04(12)$ \\
\hline $\mathrm{O}(8)-\mathrm{Mo}(4)-\mathrm{O}(6) \# 1$ & $86.13(14)$ & $\mathrm{O}(11)-\mathrm{Mo}(4)-\mathrm{O}(9)$ & $102.08(17)$ \\
\hline $\mathrm{O}(3)-\mathrm{Mo}(4)-\mathrm{O}(9)$ & $87.13(15)$ & $\mathrm{O}(8)-\mathrm{Mo}(4)-\mathrm{O}(9)$ & $153.05(12)$ \\
\hline $\mathrm{O}(6) \# 1-\mathrm{Mo}(4)-\mathrm{O}(9)$ & $86.47(15)$ & $\mathrm{O}(11)-\mathrm{Mo}(4)-\mathrm{O}(5)$ & $178.19(17)$ \\
\hline $\mathrm{O}(3)-\mathrm{MO}(4)-\mathrm{O}(5)$ & $76.89(9)$ & $\mathrm{O}(8)-\mathrm{M}(4)-\mathrm{O}(5)$ & $76.62(14)$ \\
\hline $\mathrm{O}(6) \# 1-\mathrm{Mo}(4)-\mathrm{O}(5)$ & $76.16(9)$ & $\mathrm{O}(9)-\mathrm{Mo}(4)-\mathrm{O}(5)$ & $76.45(15)$ \\
\hline $\mathrm{Mo}(1)-\mathrm{O}(2)-\mathrm{Mo}(3)$ & $115.93(16)$ & $\mathrm{Mo}(4)-\mathrm{O}(3)-\mathrm{Mo}(1)$ & $116.74(13)$ \\
\hline $\mathrm{Mo}(2)-\mathrm{O}(4)-\mathrm{Mo}(1)$ & $116.48(15)$ & $M o(1)-O(5)-M o(1) \# 1$ & $179.3(2)$ \\
\hline $\mathrm{Mo}(1)-\mathrm{O}(5)-\mathrm{Mo}(3)$ & $90.27(12)$ & $\operatorname{Mo}(1) \# 1-O(5)-M o(3)$ & $90.43(12)$ \\
\hline $\operatorname{Mo}(1)-\mathrm{O}(5)-\mathrm{Mo}(4)$ & $90.01(3)$ & $\operatorname{Mo}(1) \# 1-\mathrm{O}(5)-\mathrm{Mo}(4)$ & $89.99(3)$ \\
\hline
\end{tabular}


Table 3. continued

\begin{tabular}{llll}
\hline \hline $\mathrm{Mo}(3)-\mathrm{O}(5)-\mathrm{Mo}(4)$ & $89.98(12)$ & $\mathrm{Mo}(1)-\mathrm{O}(5)-\mathrm{Mo}(4) \# 1$ & $89.99(3)$ \\
$\mathrm{Mo}(1) \# \mathrm{l}-\mathrm{O}(5)-\mathrm{Mo}(4) \# 1$ & $90.01(3)$ & $\mathrm{Mo}(3)-\mathrm{O}(5)-\mathrm{Mo}(4) \# 1$ & $90.35(12)$ \\
$\mathrm{Mo}(4)-\mathrm{O}(5)-\mathrm{Mo}(4) \# 1$ & $179.7(2)$ & $\mathrm{Mo}(1)-\mathrm{O}(5)-\mathrm{Mo}(2)$ & $89.73(12)$ \\
$\mathrm{Mo}(1) \# 1-\mathrm{O}(5)-\mathrm{Mo}(2)$ & $89.57(12)$ & $\mathrm{Mo}(3)-\mathrm{O}(5)-\mathrm{Mo}(2)$ & 180.0 \\
$\mathrm{Mo}(4)-\mathrm{O}(5)-\mathrm{Mo}(2)$ & $90.02(12)$ & $\mathrm{Mo}(4) \# 1-\mathrm{O}(5)-\mathrm{Mo}(2)$ & $89.65(12)$ \\
$\mathrm{Mo}(1)-\mathrm{O}(6)-\mathrm{Mo}(4) \# 1$ & $117.10(14)$ & $\mathrm{Mo}(2)-\mathrm{O}(8)-\mathrm{Mo}(4)$ & $117.22(15)$ \\
$\mathrm{Mo}(3)-\mathrm{O}(9)-\mathrm{Mo}(4)$ & $116.68(16)$ & $\mathrm{C}(16)-\mathrm{N}(1)-\mathrm{C}(8)$ & $109.2(4)$ \\
$\mathrm{C}(16)-\mathrm{N}(1)-\mathrm{C}(12)$ & $108.2(4)$ & $\mathrm{C}(8)-\mathrm{N}(1)-\mathrm{C}(12)$ & $111.5(4)$ \\
$\mathrm{C}(16)-\mathrm{N}(1)-\mathrm{C}(4)$ & $111.5(4)$ & $\mathrm{C}(8)-\mathrm{N}(1)-\mathrm{C}(4)$ & $108.1(4)$ \\
$\mathrm{C}(12)-\mathrm{N}(1)-\mathrm{C}(4)$ & $108.3(4)$ & & \\
\hline
\end{tabular}

Symmetry transfomations used to generate

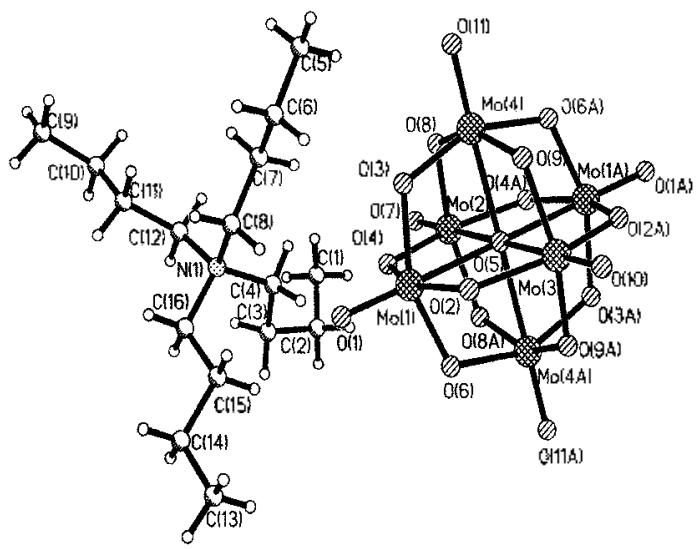

Fig. 1. Molecular structure for $\left[n-\mathrm{Bu}_{4} \mathrm{~N}\right]_{2}\left[\mathrm{Mo}_{6} \mathrm{O}_{19}\right]$ with the atomic numbering scheme.

Thermogravimetric analysis. The curves of the thermogravimetric (TG) analysis and differential thermal gravimetric (DTG) analysis for the title com-

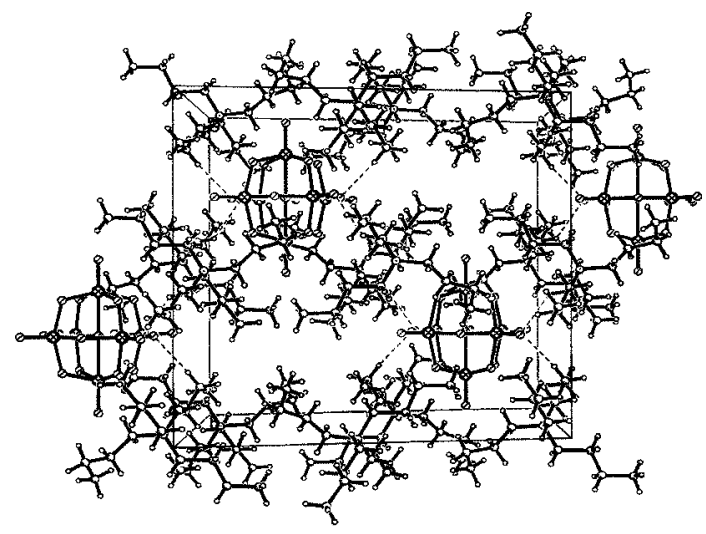

Fig. 2. $A$ view of the crystal packing down the $a$ axis for $[n-$ $\left.\left.\mathrm{Bu}_{4} \mathrm{~N}\right]_{2} \mid \mathrm{Mo}_{15} \mathrm{O}_{19}\right]$.

pound are shown in the Fig. 3. It can be seen that the thermal decomposition of the compound includes two transitions. There are two endothermic peaks,

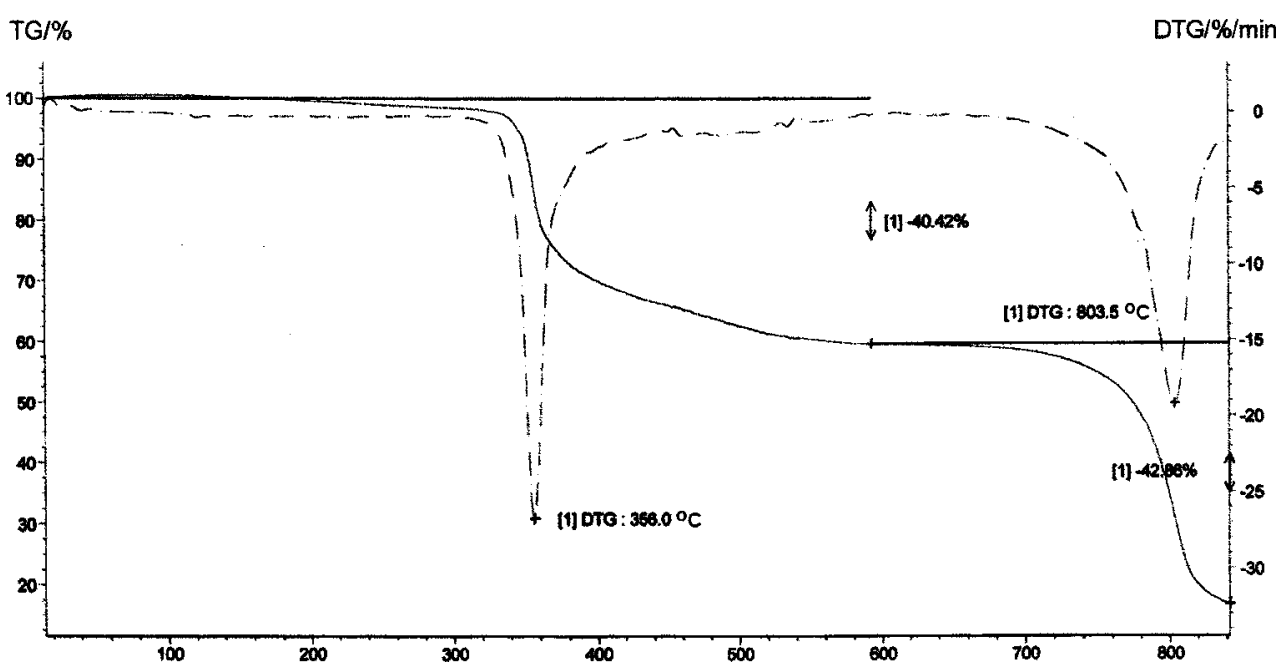

Fig. 3. TG/T'TG curves of $\left[n-\mathrm{Bu}_{4} \mathrm{~N}\right]_{\underline{i}}\left[\mathrm{Mo}_{0} \mathrm{O}{ }_{3}\right]$. 
one intense heat-absorbing peak at $356.0^{\circ} \mathrm{C}$ and the other at $803.5^{\circ} \mathrm{C}$. It shows no decomposition before $356.0^{\circ} \mathrm{C}$; but at $356.0^{\circ} \mathrm{C}$, decomposition occurs. On the base of weight changes in the TG curve, the first process of the weight loss $(40.42 \%)$ corresponds to the loss of two $\left[n-\mathrm{Bu}_{4} \mathrm{~N}\right]^{+}$groups and four oxygen atoms of $\left[\mathrm{Mo}_{6} \mathrm{O}_{19}\right]^{2}$ anions (found $40.42 \%$, calc. $40.16 \%)\left(356.0-600.0^{\circ} \mathrm{C}\right)$, with an intense endothermic phenomenon; the second process of the weight loss $(42.86 \%)$ is attributed to the further decomposition of the $\left.\mathrm{Mo}_{6} \mathrm{O}_{15}\right]$ group and the residue may be $\mathrm{Mo}_{2} \mathrm{O}_{2}$ (found $16.41 \%$, calc. $16.72 \%$ ) $\left(600.0-803.5^{\circ} \mathrm{C}\right)$. The title compound also has high thermal stability.

Acknowledgments. This work was supported by Natural Science Foundation of Shandong Province (No.Y2002B06), China.

\section{REFERENCES}

1. Ohashi, Y.; Yanagi, K.; Sasads, Y.; Yamase, T. Bull. Chem. Soc. Jpn. 1982, 55, 1254.

2. Prosser-Mccartha, C. M.; Kadkhodayan, M.; Williamson, M. M.; Bouchand, D. A.; Hill, C. L. J. Chem, Soc, Chem. Commun. 1986, 1747.

3. Willamson, M. M; Boouchard, D. A.; Hil, C. L. Inorg.
Chem. 1987, 26, 1436.

4. Hill, C. L; Bouchard, D. A.; Kadkhodayan, M.; Willamson, M. M.; Schmidt, J. A.; Hilinski, E. F. J. Am. Chem. Soc. 1988, /10, 5471.

5. Attanasio, D.; Bonamico, M.; Fares, V; Imperatori, P.; Suber, L. J. Chem, Soc., Dalton Trans. 1990, 3221.

6. Attanasio, D.; Bonamico, M.; Fares, V; Sube, L. J. Chem. Soc., Dalton Trans, 1992, 2523.

7. Xu, X. X.; You, X. Z.; Wang, X. Polyhedron, 1994, 13, 1011.

8. Pope, M. T.; Muller, A. Angew. Chem. Int. Ed. Eng. 1991, 30, 34.

9. Launary, J. P. J. Inorg. Nucl. Chem. 1976, 38, 807.

10. Xu, X. X.; You, X. Z.; Wang, X. Acta Chemica Scandinavica. 1995, 5 .

11. Sheldrick, G. M. Actc Cryst., Sect. A 1969, 46, 467.

12. Sheidrick, G. M. SHELXTL97, Program for Crystal Strucnure refinement, University of Gottingen, Germany, 1993.

13. Wilson. A. J. International Table for X-ray Crystallography, volume C, 1992; Kluwer Academic Publishers, Dordrecht: Tables 6.1.1.4 (pp. 500-502) and 4.2.6.8 (pp. 219-222) respectively.

14. Fuchs, S.; Fretwald, W.; Hartl, H. Acta Crystallogr., Sect. B. 1978, 34, 1764.

15. Leegg, W.; Sheldrick, G. M. Acta Crystallogr., Sect. B. 1982, 2906.

16. Steiner Th. Cryst. Rev, 1996, 6, 1.

17. Jeffrey, G. A.; Maluszynska, H.; Mitra J.; Int. J. Biol. Macromol, 1985, 7, 336. 